\title{
Correction: Oh, J.Y., et al. Synergistic Autophagy Effect of miR-212-3p in Zoledronic Acid-Treated In Vitro and Orthotopic In Vivo Models and in Patient-Derived Osteosarcoma Cells, Cancers 2019, 11, 1812
}

Ju Yeon Oh ${ }^{1,2,+}$, Eun Ho Kim ${ }^{3,+}$, Yeon-Joo Lee ${ }^{4} \mathbb{D}$, Sei Sai ${ }^{5}$, Sun Ha Lim ${ }^{3}$, Jang Woo Park ${ }^{6}$, Hye Kyung Chung ${ }^{6}$, Joon Kim ${ }^{1}$, Guillaume Vares ${ }^{7}{ }^{\mathbb{D}}$, Akihisa Takahashi ${ }^{8}{ }^{\mathbb{D}}$, Youn Kyoung Jeong ${ }^{9}$, Mi-Sook Kim ${ }^{10, *}$ and Chang-Bae Kong ${ }^{11, *}$

1 Laboratory of Biochemistry, Division of Life Sciences, Korea University, Seongbuk-gu, Seoul 02841, Korea; ojo5295@kirams.re.kr (J.Y.O.); joonkim@korea.ac.kr (J.K.)

2 Division of Radiological Science and Clinical Translational Research, Korea Cancer Center Hospital, Nowon-gu, Seoul 01812, Korea

3 Department of Biochemistry, School of Medicine, Daegu Catholic University, Nam-gu, Daegu 42472, Korea; eh140149@cu.ac.kr (E.H.K.); sunha112@cu.ac.kr (S.H.L.)

4 Division of Radiation Biomedical Research, Korea Institute of Radiological and Medical Sciences, Seoul 01812, Korea; eyeonjoo@gmail.com

5 Department of Basic Medical Sciences for Radiation Damages, National Institute of Radiological Sciences, Chiba 263-8555, Japan; sai.sei@qst.go.jp

6 Korea Drug Development Platform Using Radio-Isotope, Korea Institute of Radiological \& Medical Sciences, Seoul 139-706, Korea; jangwoo@kirams.re.kr (J.W.P.); hkchung@kirams.re.kr (H.K.C.)

7 Cell Signal Unit, Okinawa Institute of Science and Technology Graduate University (OIST), Okinawa 1919-1, Japan; guillaume.vares@oist.jp

8 Gunma University Heavy Ion Medical Center, Maebashi 371-8511, Gunma, Japan; a-takahashi@gunma-u.ac.jp

9 Research Center for Radiotherapy, Korea Institute of Radiological and Medical Sciences, Seoul 139-706, Korea; amy3523@kirams.re.kr

10 Department of Radiation Oncology, Korea Institute of Radiological and Medical Sciences, Seoul 139-706, Korea

11 Department of Orthopaedic Surgery, Korea Institute of Radiological and Medical Sciences, Seoul 139-706, Korea

* Correspondence: eh140149@naver.com or mskim@kirams.re.kr (M.-S.K.); cbkongmd@gmail.com (C.-B.K.)

+ These authors contributed equally to this work.

Received: 10 April 2020; Accepted: 15 May 2020; Published: 26 May 2020

The authors wish to make the following corrections to this paper [1]:

The authors would like to replace Figure $3 b$ in [1]. The correction is to correct errors in the arrangement of the Western blots. The original version of Figure $3 \mathrm{~b}$ is: 
b.

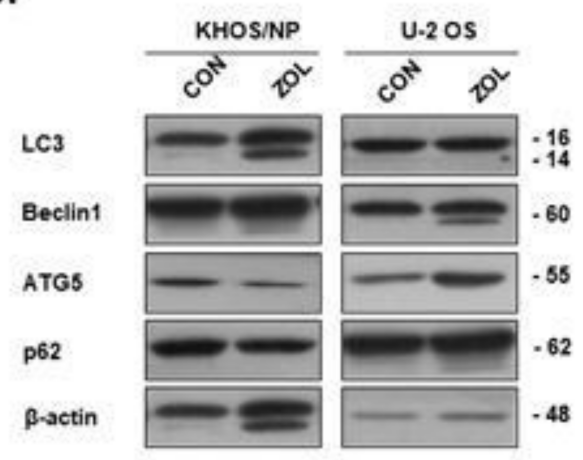

and should be replaced with the following Figure $3 b$ :

b.

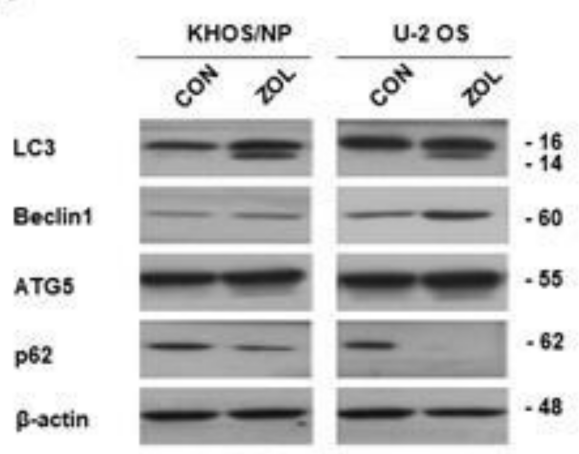

The authors would like to replace the first affiliation in [1]. The original affiliation is "Laboratory of Biochemistry, School of Life Sciences and Biotechnology, Korea University, Seongbuk-gu, Seoul 136-701, Korea" and should be replaced with "Laboratory of Biochemistry, Division of Life Sciences, Korea University, Seongbuk-gu, Seoul 02841, Korea".

The authors would like to apologize for any inconvenience caused to the readers by these changes.

\section{References}

1. Oh, J.Y.; Kim, E.H.; Lee, Y.J.; Sai, S.; Lim, S.H.; Park, J.W.; Chung, H.K.; Kim, J.; Vares, G.; Takahashi, A.; et al. Synergistic Autophagy Effect of miR-212-3p in Zoledronic Acid-Treated In Vitro and Orthotopic In Vivo Models and in Patient-Derived Osteosarcoma Cells. Cancers 2019, 11, 1812. [CrossRef] [PubMed] 